\title{
Charged Particle Beam Current Monitoring Tutorial
}

\author{
Robert C. Webber \\ Fermi National Accelerator Laboratory \\ P.O. Box 500, Batavia, Illinois 60510
}

October 1994

Presented at the Beam Instrumentation Workshop, Vancouver, Canada, October 3-6, 1994 


\section{Disclaimer}

This report was prepared as an account of work sponsored by an agency of the United States Government. Neither the United States Government nor any agency thereof, nor any of their employees, makes any warranty, express or implied, or assumes any legal liability or responsibility for the accuracy, completeness, or usefulness of any information, apparatus, product, or process disclosed, or represents that its use would not infringe privately owned rights. Reference herein to any specific commercial product, process, or service by trade name, trademark, manufacturer, or otherwise, does not necessarily constitute or imply its endorsement, recommendation, or favoring by the United States Government or any agency thereof. The views and opinions of authors expressed herein do not necessarily state or reflect those of the United States Government or any agency thereof. 


\title{
Charged Particle Beam Current Monitoring Tutorial
}

\author{
Robert C. Webber \\ Fermi National Accelerator Laboratory*, P.O. Box 500, Batavia, Illinois 60510 USA
}

\begin{abstract}
A tutorial presentation is made on topics related to the measurement of charged particle beam currents. The fundamental physics of electricity and magnetism pertinent to the problem is reviewed. The physics is presented with a stress on its interpretation from an electrical circuit theory point of view. The operation of devices including video pulse current transformers, direct current transformers, and gigahertz bandwidth wall current style transformers is described. Design examples are given for each of these types of devices. Sensitivity, frequency response, and physical environment are typical parameters which influence the design of these instruments in any particular application. Practical engineering considerations, potential pitfalls, and performance limitations are discussed.
\end{abstract}

\section{INTRODUCTION}

Particle accelerators are constructed and operated for a growing variety of applications from high energy physics research to cancer treatment, isotope production, biological studies, lithography, and even food preservation. One fundamental measure of an accelerator's or beam line's performance and a necessary parameter in the application of it's particle beam is the quantity or intensity of particles accelerated and transported to the final point of utilization.

Intensity measurements may be made with intercepting or non-intercepting monitors. Intercepting monitors operate by introducing sufficiently dense material directly into the particle beam's path to produce measurable signals from the resulting ionization or nuclear processes. Non-intercepting monitors rely on macroscopic scale interaction with the electromagnetic fields of the beam for signal energy.

Intercepting devices are not necessarily destructive of the beam being measured, but most implementations result in absorption of a large amount of energy from the beam particles or, in the case of ion beams, a change of the charge state. A large variety of intercepting devices exist and are used in applications where these side-effects are acceptable, most often in single pass situations where the beam particles each pass through the device only once. Intercepting monitors have the advantage that they may be designed to measure neutrally charged particle beams.

* Work supported by the U.S. Department of Energy under contract No. DE-AC02-76CH03000. 
Intercepting monitors are rarely acceptable in applications, such as synchrotrons, where each particle of the circulating beam passes through a monitoring device many times. The utilization of non-intercepting instruments, sensitive to the electromagnetic fields due to the particles' charge and its motion, is quite natural, since the accelerators themselves rely on interactions with those same fields for acceleration and steering. The flow of charged particles through an accelerator or beam line constitutes, in the strictest sense, an electric current, proportional to the number of particles and to the charge carried by each. As a result non-intercepting beam intensity instruments are often referred to as beam current monitors. They exhibit same properties and are designed on the same principles as current monitors in the electrical circuit sense.

This paper shall focus on monitors of the non-intercepting type. Even among these, the diversity of designs and performance parameters is large. $(1,2,3)$ Different applications demand devices covering a broad range of current sensitivities and time or frequency response. Current magnitudes to be measured vary from kiloamperes in induction linacs to microamperes or less in heavy ion accelerators. The wide range of time responses necessary is indicated by application examples including measurement of low duty cycle picosecond current pulses in electron machines like LEP, high duty cycle nanosecond pulses in hadron accelerators like the Tevatron in fixed target mode, video frequency pulses like a macropulse of beam through a proton linac, and the direct current component of circulating beam in storage rings and colliders. Frequently a single machine needs instruments to measure several of these extremes. For example, the Fermilab Tevatron, in collider mode, requires high accuracy and high stability dc current measurement for quick determination of beam storage lifetime, as well as fast and accurate measurement of individual nanosecond bunch intensities to differentiate between proton and antiproton bunches.

\section{FUNDAMENTALS}

Associated with the particle beam are electric and magnetic fields, due respectively to the charge carried by the particles and the fact that the charges are in motion. Coupling to either one or a combination of both these fields will provide a signal related to the beam intensity. Devices which couple primarily to the electric field are occasionally used to measure high frequency beam signal components for intensity monitoring purposes. It is difficult, however, to obtain satisfactory low frequency response with such capacitive sensors without resorting to high impedance circuitry which can lead to noise susceptibility. As will be shown later, a severe penalty, in terms of available signal power, may be incurred with the use of these electric field devices, especially in the video frequency band. The discussion in this article will mainly concentrate on utilization of the beam's magnetic field as a signal source. 


\section{A Particle Beam as an Electric Current}

In the purest sense, a beam of charged particles in motion is an electric current. The magnitude of that current is simply

$$
I_{b}=q \lambda_{N} v
$$

where $q$ is the beam particle's charge, $\lambda_{N}$ the number of particles per unit length, and $v$ the velocity at which the particles are traveling. In a circuit sense, the beam appears as an ideal current source manifesting a nearly infinite source impedance. That this is the case can be readily seen by considering that source impedance is simply that change in voltage which must be applied to the source to force a given change in current at its terminals. Since the beam current is determined by the velocity of the charged beam particles, a large enough voltage must be applied to significantly alter this velocity in order to cause any change in current. For all but the lowest energy beam, this must typically be a voltage comparable to the beam's energy (in $\mathrm{eV}$ units). Clearly the impedance of the beam is normally extremely large.

This should not be construed to imply however that all beam sensors exhibit a large source impedance. Any device constructed has inherent shunting impedances which contribute source impedance terms as observed by an external circuit. The beam itself simply contributes nothing to this impedance. The simplest electric field sensing devices (capacitive probes or pick-ups) will exhibit a capacitive source impedance equal to the capacitance between the electrode and its surroundings. Magnetic type pick-ups in their simplest design present an inductive source impedance.

A particle beam usually has a complex spatial charge distribution along the axis of motion, resulting in a current with a broad frequency spectrum. The typical example is a pulsed or periodic large scale longitudinal distribution comprised on a finer scale of multiple individual bunches. In a synchrotron or storage ring this pulse is periodic with the revolution frequency resulting in signal components from dc to frequencies corresponding to the inverse of the bunch length. A useful reference on frequency domain analysis of bunched beam signals has been written by Siemann.(4) A rare example of a beam with very few ac current components is the debunched anti-proton beam stored in the Fermilab accumulator ring where particles uniformly fill the circumference. Nevertheless, the discrete particle nature of the beam results in wideband shot noise current components which are the source of the signals essential for beam emittance reduction by stochastic cooling in that machine.

A dc beam current component, that is, a net charge transfer in one direction via the beam, will nearly always be present. This must be the case in a beam transport system with any magnetic bending elements. Such a system will correctly steer and transport only beams with one sign of current, i.e. particles of opposite charge 
can both travel through that system only if they travel in opposite directions. A positive charge to the right and a negative charge to the left both appear as current of the same sign. In the Fermilab Tevatron, for example, the clockwise circulating protons and the counter clockwise circulating antiprotons each produce signals which add with the same sign in a dc current monitor. To cancel each other's dc component both would need to travel the same direction which is not possible.

The rich spectrum of the typical beam currents provides both advantages and potential problems. The availability of signal nearly anywhere in the spectrum permits much design flexibility and a wide range of options for current monitoring instruments. However, all spectral components outside the design bandwidth of an instrument represent potential noise sources. These signals can feed through to the output via unsuspected resonances, by overloading active circuitry, by aliasing within sampled signal type circuits, or by any other non-linear process. It is quite easy, for example, to construct a dc current monitor that will indicate the presence of a dc current component in response to only an ac stimulation. Carelessness in understanding and controlling an instrument's response to all frequencies to which it may be exposed can lead to unexpected and difficult to interpret output signals. Even for in-band signals, it is often necessary to understand the relationship between the average beam current and the charge distribution responsible for that current. This is especially true in the case of narrowband monitors. The signal observed at the fundamental frequency for a given amount of charge distributed with $100 \%$ sinusoidal modulation will be one amplitude; that for the same quantity of charge distributed as a train of delta functions with the same periodicity will be two times that amplitude. This follows simply from the Fourier decomposition of the distributions.

In the consideration of time and frequency aspects of beam current monitoring, an interesting observation presents itself in proton accelerators (e.g. Fermilab Linac and Booster) where the range of energies is non-relativistic and the particle velocity increases as the beam is accelerated. Down the Linac, even though particle velocity is a strong function of location, each beam current monitor (assuming no lost particles) is observed to read the same current as all others in the Linac. Yet in the Booster as the particle velocity increases a current monitor indicates a proportionally increasing current.

How is this paradox explained? The Linac is arranged to function as a fixed frequency system. The spacing between accelerating cells is adjusted to be proportional to the velocity corresponding to the particle energy at each point in the Linac. This results in a constant transit time between cells to match the fixed if accelerating frequency. Physically the beam is a group of bunches each containing some constant charge and always separated in time by the cell to cell transit time (or some fixed multiple.) The beam current averaged over the time interval between bunches is simply that bunch charge divided by the interval. This is a conserved quantity everywhere in the Linac and all monitors read the same current. Consistency is maintained with Eq. 1 because as the velocity increases $\lambda_{N}$, 
the number of particles per unit length, actually decreases! The spatial charge distribution is stretched as the beam progresses through the Linac. You can convince yourself of this by considering the spacing between two automobiles (with the same initial velocity) before and after the acceleration of coasting down a hill on the roadway.

In the Booster synchrotron, the beam path length around the machine remains closely fixed while the particle velocity increases by about $50 \%$. If the total charge in the ring is constant but passes any current monitor location once per decreasing revolution period, then the current observed at that location indeed increases with the particle velocity. To first order, the spatial charge distribution in a synchrotron is preserved as the revolution frequency increases. This increasing beam current effect becomes negligible as the particles become highly relativistic and the limiting velocity is reached.

\section{The Magnetic Field and Magnetic Induction}

The magnetic field produced by the beam current is the source of signal energy for the current monitor. The magnitude of this field in the vicinity of the particle beam in a vacuum is calculated using Ampere's Law

$$
\oint(\boldsymbol{B} \cdot d \boldsymbol{l})=\mu i
$$

in the same manner as elementary text books derive the field around a wire.(5) The result is that the magnitude of $B$ at a distance $r$ from the beam is

$$
B(r)=\mu \frac{i}{2 \pi r}
$$

with the lines of induction forming concentric circles around the beam. For our purposes the temporal variations of $B$ can be taken as identical to those of the current itself. For every spectral component of the current, there is a corresponding spectral component of $B$, including dc.

Magnetic induction provides the necessary coupling between the beam and the signal pick-up in the classical current monitor. Faraday's Law of Induction

$$
\oint(E \cdot d l)=-\frac{d \Phi_{B}}{d t}
$$

where $\Phi_{B}=\int B \cdot d S$ is the magnetic flux due to the beam current, provides the basis for design of magnetic sensors and allows calculation of available signal. Faraday's Law simply states that the induced emf around any path, e.g. a loop of wire, is proportional to the time rate of change of magnetic flux through the area 
enclosed by that path. Note that a simple induction loop can provide no information as to the dc component of the magnetic field and therefore of the dc beam current since that flux contribution has zero time derivative.

In circular accelerators or storage rings, the beam travels around the circumference forming an approximately circular current loop. Such a loop is a classical magnetic dipole with lines of induction cutting normal to the plane of the loop.(Fig. 1a) Accelerator beams, of course, travel through evacuated regions of space, typically enclosed by metallic tubes. In the simplest geometry the beam current loop is concentrically enclosed by a conducting beam tube. This seems quite natural and benign from a vacuum and mechanical viewpoint. However, when subjected to inspection from an electrical point of view, it is observed that the beam tube forms a 'pick-up loop' intercepting nearly all the beam induced flux penetrating the ring's center area. The beam and its vacuum tube form a transformer of the simplest design with the beam acting as the primary 'winding'

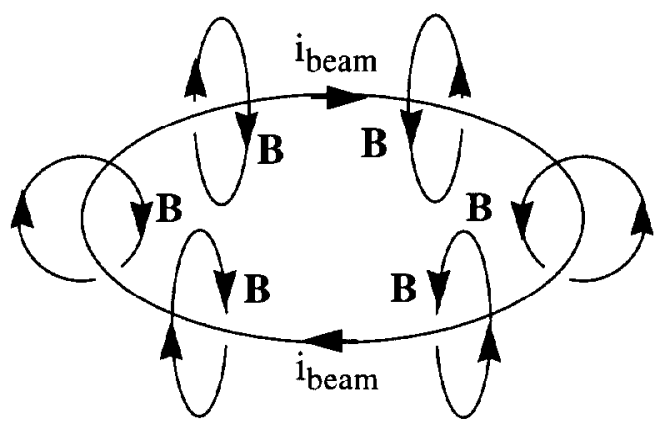

(a)

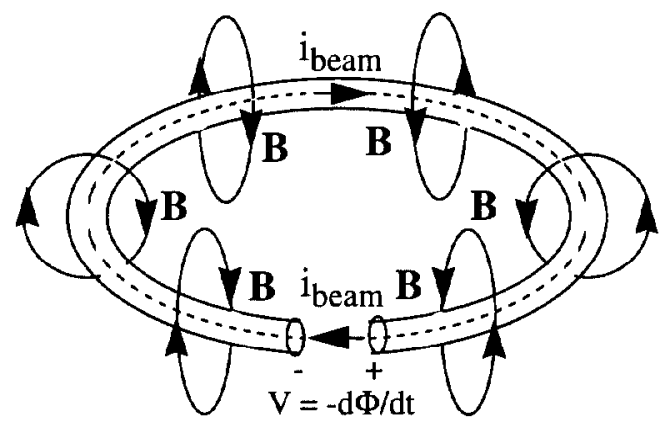

(c)

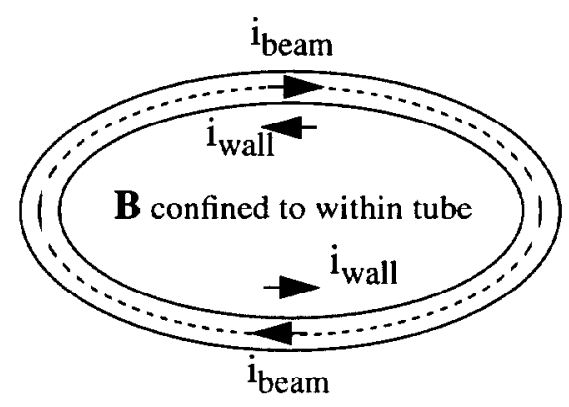

(b)

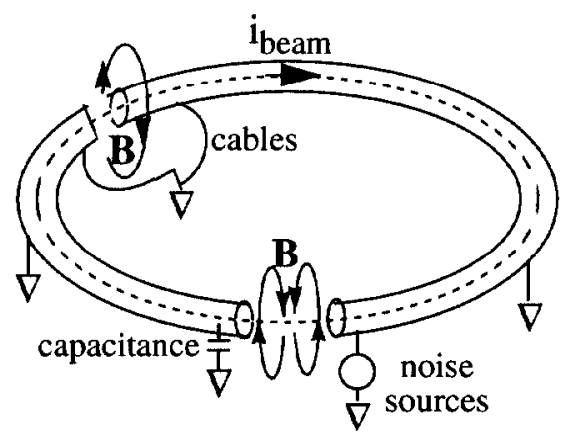

(d)

Figure 1. (a) Lines of magnetic induction around circulating beam. (b) Wall currents induced in beam tube attenuate external B. (c) Break in tube impedes wall currents permitting external $\mathbf{B}$ and appearance of induced voltage. (d) Layout of typical accelerator shows complex and distributed paths available to induced currents. 
and the tube, the secondary. In this manner, so called 'wall currents' are induced in the beam tube when the secondary circuit is completed.(Fig. 1b)

Lenz's Law, in one form, states that the induced currents will flow in the opposite sense of the current responsible for their induction. Applied in this case it means that the wall currents flow opposed to the beam current. These currents produce flux of their own with a sign so as to reduce the primary (beam) current's flux within the enclosed loop area. This effectively 'shields' the space outside the beam tube from the beam's magnetic fields. To the extent that the wall current and the beam current may be equal in magnitude this shielding is perfect and the beam's field is confined within the tube.

Attempts to measure the beam current using an inductive pickup loop outside the beam tube become futile due to this flux cancellation effect. At audio frequencies this can be overcome by inserting a short non-conducting section in the beam tube to interrupt the induced wall current and inhibit the flux cancellation outside the beam tube.(Fig. 1c) The voltage induced across such a gap can and indeed has been be used as a beam monitor. The typical accelerator however is not so simple. The vacuum chamber contains kicker magnets, accelerating cavities, and numerous dc breaks for various reasons (like someone else's beam monitor). In addition, the beam tube is generally grounded, intentionally or by chance, at numerous locations forming shunt current paths around the gap. At even moderate frequencies, the effects of capacitance to ground and across the gap become significant. These effects tend to 'localize' the induction current paths with the result that what is happening on the other side of the ring is of little matter and it is not useful to think of the beam tube as a simple global induction loop.(Fig. 1d)

At high frequencies the skin effect constrains all beam induced currents to the inner wall of the beam tube. At frequencies where the skin depth becomes small compared to the tube wall thickness the magnetic shielding effect of the tube becomes nearly perfect and only severely attenuated magnetic information about the beam current exits outside the beam tube. However, any break in the pipe will interrupt even these high frequencies and act as a 'window' permitting the high frequency currents and their associated fields to 'leak' outside. This situation is frequently used to advantage, and introduced by design, in current monitors, if cavities, etc. Unfortunately the same 'leaks' occur at breaks introduced by chance or carelessness and become a source of noise to un-shielded circuits.

No dc or zero frequency component is permitted by Faraday's Law to exist in the wall current: That component of the beam's field penetrates the beam tube unaffected (provided the tube is not made of a magnetic material.) Complete information of the beam's direct current component exists external to the beam tube, though no simple inductive loop can detect it. Slightly more complicated devices, such as the DCT described later, can serve effectively in this situation.

All beam current monitors operate on the principal of intercepting lines of magnetic induction produced by the beam current. Any successful design must have access to some component of that magnetic flux and must take complete 
account of the environment determining the distribution of that flux in the sensitive volume of the monitor. All resistive and reactive shunting current paths, all shunting magnetic paths, and all external field sources (magnetic and electric) must be controlled or the consequences of unpredictable performance may be suffered.

\section{BEAM CURRENT MONITOR DESIGNS}

The commonly used beam current monitor configurations fall into four general categories: classical ac transformers, wall current monitors, dc monitors, and narrowband if monitors.

Toroidal transformers and variations thereof are generally used for monitoring beam currents in the audio to tens of megahertz frequency range. To intercept the heam's magnetic flux, these transformers are built within the metal vacuum housing or around a dc break in the beam pipe. Electrostatic shielding is generally included to prevent unwanted signals due to capacitive coupling to the beam charge.

A special variation of the toroidal transformer called a wall current monitor is used for wide bandwidth current measurements up to a few gigahertz. It can be thought of as a device which produces a signal by directly intercepting the induced wall currents. Since it is designed to function at high frequencies, it requires access to the inner wall of the beam tube either directly or through an appropriate 'window.'

DC beam current monitors, though having access to the beam's dc magnetic field even outside a conducting beam tube, are generally built around a break in the tube to permit response to audio and higher frequency signal components. Practical dc monitors are hybrid instruments combining a dc sensitive magnetic modulator section and a classical ac transformer to offer moderately high bandwidth with dc response.

Narrow band current monitors are designed to be sensitive to selected spectral components of the beam current signal. Devices of this type can offer the high transfer impedance necessary for measurement of small beam currents. These monitors for begin to look like accelerating cavities, though they are not generally required to handle high power. They can operate within and as an inherent part the vacuum vessel or external to it with an appropriate 'window.'

\section{Classical AC Transformers}

The classical ac transformer consists of a primary and one or more secondary windings typically on a toroidal form as depicted in Fig. 2a. Faraday's Law gives 
the voltage on any winding as

$$
V_{k}=-N_{k} \frac{d \Phi_{T}}{d t}
$$

where $\Phi_{\mathrm{T}}$ is the total flux linking that winding.

The contribution to the total flux within the toroid due to a wire current $i$ flowing in any $N$ turn winding $k$ is

$$
\Phi_{k}=\int \boldsymbol{B}_{k} \cdot d \boldsymbol{S}=\int_{a}^{b} \mu \frac{N_{k} i_{k}}{2 \pi r} h d r=\mu \frac{N_{k} i_{k} h}{2 \pi} \ln \frac{b}{a} .
$$

This follows from the definition of flux and Eq. 3. It is customary and often more convenient from a circuit viewpoint, rather than applying the flux concept directly, to use the quantity known as self inductance, $L$. The relationship between the self inductance of winding $k$ and its associated flux is defined by

$$
\left(V_{k}=-N_{k} \frac{d \Phi}{d t}=L_{k} \frac{d i_{k}}{d t}\right) \Rightarrow\left(L_{k}=\frac{-N_{k} \Phi_{k}}{i_{k}}\right) .
$$

Combining this with Eqs. 6 yields

$$
L_{k}=\mu \frac{N_{k}^{2} h}{2 \pi} \ln \frac{b}{a}=N_{k}^{2} L_{0} \approx \mu \frac{N_{k}^{2} A}{l}
$$

where $L_{0}=(\mu h / 2 \pi) \ln (b / a)$ is simply the single-turn inductance. This reveals that inductance is purely a property of the geometry of the winding, the number of turns, and the relative permeability of the toroid core. The final approximate form of Eq. 8 is valid for a thin-walled toroid geometry, where $\boldsymbol{B}$ can

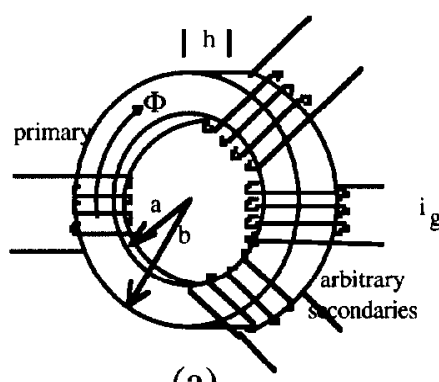

(a)

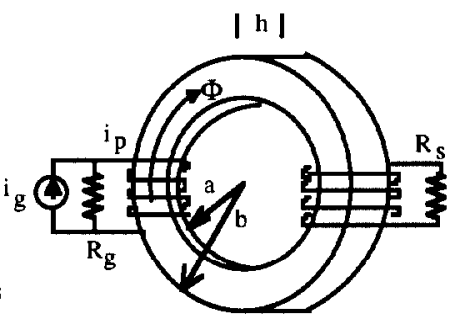

(b)

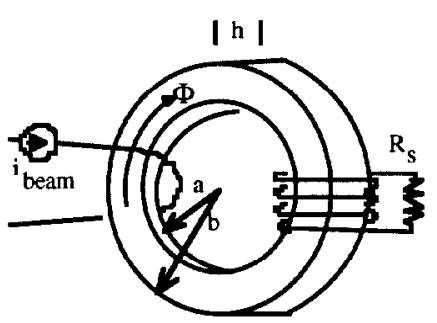

(c)

Figure 2. (a) Generic toroidal transformer. (b) Typical two winding current transformer. (c) Beam current transformer. 
be taken as constant between radii $a$ and $b . A$ is the cross-sectional area of the toroid, $A=(b-a) h$, and $l$ is the magnetic path length around the toroid, $l=\pi(b+a)$. This form is handy in transformer design since magnetic material vendors frequently specify their toroid products in terms of effective cross section and effective magnetic length.

Given these fundamentals, the performance of a transformer in any circuit configuration may be calculated. Assuming all windings link the total flux in the toroidal core, that flux is simply the sum of the contributions from all windings:

$$
\Phi_{T}=\sum_{m=1}^{k} \Phi_{m}=\sum_{m=1}^{k} \frac{L_{m} i_{m}}{N_{m}} .
$$

Solving simultaneously with Eq. 5 for all windings, the current and voltage solutions for all coupled circuits may be obtained.

Applying this to the configuration in Fig. 2b, find

$$
\Phi_{T}=\frac{L_{p} i_{p}}{N_{p}}+\frac{L_{s} i_{s}}{N_{s}}
$$

and

$$
\frac{d \Phi_{T}}{d t}=-\frac{V_{p}}{N_{p}}=-\frac{V_{s}}{N_{s}} .
$$

At this point it is convenient to equate the $N$ turn primary to the single turn beam, $i_{p}=i_{b}$ and $N_{p}=1$, (Fig. 2c) and to be satisfied with the steady state frequency domain solution permitting replacement of the derivative operator with the LaPlace variable $s=j \omega$. Given $i_{s}=V_{s} / R_{s}$, Eqs. 10 and 11 respectively become

$$
\begin{gathered}
\Phi_{T}=L_{0} i_{b}+\frac{L_{s} i_{s}}{N_{s}} \\
s \Phi_{T}=-\frac{i_{s} R_{s}}{N_{s}} .
\end{gathered}
$$

Simultaneous solution yields

$$
i_{s}=\frac{(s \tau)}{(1+s \tau)}\left(\frac{i_{b}}{N_{s}}\right)
$$


where $\tau=L_{s} / R_{s}$. This is the response of the simple beam current transformer.

Inspection of this result reveals that for frequencies $\omega \gg 1 / \tau$ the secondary winding current is simply $i_{s}=i_{b} / N_{s}$, equal in magnitude to the beam current divided by the number of turns and in phase with it. This is the characteristic of an ideal current transformer. For frequencies $\omega \ll 1 / \tau$, the secondary current is $i_{s}=(s \tau) i_{b} / N_{s}$, proportional in magnitude to the derivative (that is, the frequency) of the beam current and $90^{\circ}$ out of phase. Operation in this regime is that of the so-called B-dot coil, a small pick-up loop often used to measure the field in pulsed magnets. Note that only the ratio of $L$ and $R$ determine which regime and 'way of thinking' apply for any given signal frequency.

Available signal power is another important aspect of current monitors. Signal power determines the ability of the signal to compete with noise sources. To achieve operation in the ideal current transformer mode for all frequencies of interest set $\tau>1 / \omega_{\min }$, where $\omega_{\min }$ is the lowest signal frequency. The available signal power is then

$$
P_{\text {signal }}=i_{s}^{2} R_{s}=\frac{i_{b}^{2}}{N_{s}^{2}} R_{s}
$$

Normalizing to $i_{s}^{2}$ and substituting $N_{s}^{2} L_{0} / \tau$ for $R_{s}$ find:

$$
\frac{P_{\text {signal }}}{i_{s}^{2}}=\frac{L_{0}}{\tau}=\frac{\left(\mu h \ln \frac{b}{a}\right)}{2 \pi \tau} \approx \mu \frac{A}{l} .
$$

Available signal power is proportional to the permeability of the media and the toroid's cross-sectional area, and inversely proportional to the toroid's effective radius. This is the reason low loss, high permeability cores are commonplace in current transformer designs. Subject to the constraint that $L_{s} / R_{s} \gg 1 / \omega_{\min }$, available signal power is independent of the load resistance and the number turns.

Energy loss in the core material and the effects of capacitance between the winding turns and to ground limit the high frequency performance of classical ac transformers. Proper selection of core material and careful construction techniques are required to achieve optimal performance. Resonant responses may be damped by the use of distributed shunt resistances across the winding. These damping resistors absorb some of the available signal power, but can provide the benefit of serving as a back termination when a long cable is to be driven. Metallic shielding around the signal winding is appropriate to avoid signal contamination by 
capacitive coupling to the beam or external noise sources. The shielding or housing must be constructed so as not to act as a shorted secondary winding.

A monitor to quantify the charge content of very short, isolated beam bunches has been described by Unser. $(6,7)$ Named the integrating current transformer, this device relies on the impulse response of a modified toroidal transformer to produce an output proportional to the bunch charge. All information on the temporal characteristics of the bunch is lost.

\section{A Signal Power Comparison: Magnetic vs. Capacitive Pick-ups}

Now that the ground work is laid for a quantitative understanding of magnetic sensors, a digression is in order to appreciate the prevalence of such monitors over electric field devices.

Imagine a beam tube allowing a limited cylindrical volume of space for a beam monitor, either capacitive or magnetic. The toroidal current monitor just discussed serves as the magnetic monitor when the available volume is described by ID and OD dimensions $2 a$ and $2 b$ respectively and by axial height $h$. The corresponding capacitive sensor takes the form of a thin-walled conducting cylinder of length $h$ and radius $a$ inside a beam tube of radius $b$. (Fig. 3)

For relativistic beam particles, where all electric field components are perpendicular to the axis of motion, the capacitive electrode will intercept only and all the electric field lines of those beam particles within its length. A charge, equal and opposite to that of the contained beam, is induced on the inner surface of the

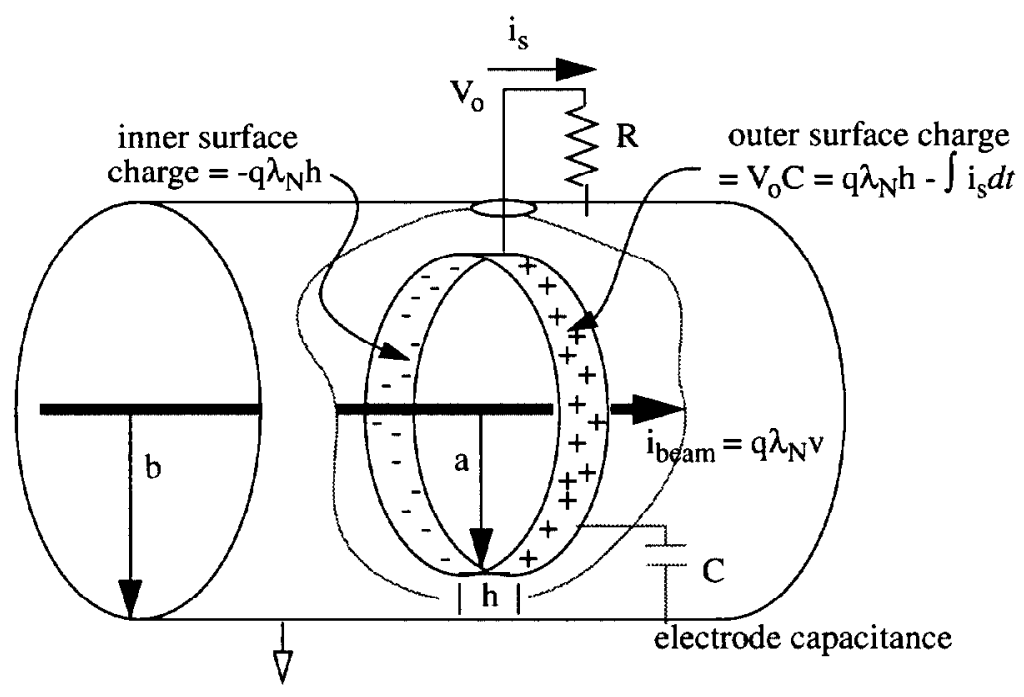

Figure 3. Capacitive beam current monitor. 
electrode. Using the variable definitions given for Eq. 1, this quantity of charge (for constant $\lambda_{N}$ within the electrode length) is

$$
q_{e b}=-\int_{-\frac{h}{2}}^{\frac{h}{2}} q \lambda_{N} d l=-q \lambda_{N} h
$$

An equal and opposite charge is induced on the outside wall of the electrode. This is the charge available to be drained off as $i_{s}$ through an external load resistance $R_{s}$ to provide signal power. The charge available on the electrode is given by

$$
q_{e}=-q_{e b}-\int_{-\infty}^{\infty} i_{s} d t .
$$

The electrode capacitance for this coaxial geometry is

$$
C=\frac{q_{e}}{V}=\frac{q_{e}}{\int_{a}^{b} E(r) d r}
$$

where $E(r)$ is found from Gauss' Law

$$
\oint(\boldsymbol{E} \cdot d \boldsymbol{S})=\frac{q_{e}}{\varepsilon}
$$

to be $E(r)=q_{e} /(2 \pi \varepsilon r h)$. The resulting capacitance is

$$
C=\frac{2 \pi \varepsilon h}{\ln (b / a)}
$$

This capacitance, in combination with the load resistance, forms an RC circuit driven by the beam induced charge currents with a signal voltage $V=q_{e} / C=i_{s} R_{s}$. Making substitutions into Eq. 18 and switching to LaPlace notation for the integral yields

$$
i_{s} C R_{s}=q \lambda_{N} h-\frac{i_{s}}{s} .
$$

Solving for $i_{s}$ with $i_{b}=q \lambda_{N} v$ leads to:

$$
i_{s}=\frac{s i_{b} h}{v(1+s \tau)}
$$


where $\tau=R_{s} C$.

As with the magnetic current monitor, this response becomes frequency independent for frequencies $\omega \gg 1 / \tau$. Under this condition $i_{s}=i_{b} h / v \tau$ and the available signal power is

$$
P_{\text {signal }}=i_{s}^{2} R_{s}=\left(\frac{i_{b} h}{v}\right)^{2} \frac{R_{s}}{\tau^{2}}=\left(\frac{i_{b} h}{v}\right)^{2} \frac{1}{\tau C}
$$

Normalizing to $i_{b}^{2}$ and substituting Eq. 21 for $C$ results in:

$$
\frac{P_{\text {signal }}}{i_{b}^{2}}=\frac{h \ln \frac{b}{a}}{2 \pi \varepsilon \tau v^{2}} .
$$

This can now be compared to the signal power available from the corresponding magnetic sensing current monitor with the same time constant, Eq. 16. The ratio of available power between the monitors is

$$
\frac{P_{M}}{P_{C}}=\varepsilon \mu v^{2}=\varepsilon \mu \beta^{2} c^{2}
$$

where $\beta=v / c$.

Noting that $\mu=\mu_{0} \mu_{r}, \varepsilon=\varepsilon_{0} \varepsilon_{r}$ and $c^{2}=1 / \mu_{0} \varepsilon_{0}$, this becomes simply

$$
\frac{P_{M}}{P_{C}}=\varepsilon_{r} \mu_{r} \beta^{2} .
$$

For relativistic beams, $\beta \approx 1$, two rather remarkable observations follow this result: 1) the available signal power is identical for either type of device where vacuum is the only media, 2) for any medium other than vacuum the magnetic monitor offers superior performance. The advantage is often orders of magnitude at moderate frequencies where relative permeabilities $>10,000$ can readily be obtained. This justifies the prevalence of magnetic type beam current monitors. Capacitive monitors, in this regime, have their place where signal power is not an important concern and at high frequencies where most magnetic materials no longer exhibit high permeability and low loss.

Eq. 27 indicates that the magnetic monitor loses its advantage for low energy beams. For example, it predicts, for a beam of $\beta=0.01$, that the signal power from a capacitive monitor will be equal to that from a magnetic monitor with a core of permeability 10,000 . Indeed, if the particles are at rest, the beam current is zero and a magnetic current transformer measures exactly that. However, caution 
is necessary in the quantitative interpretation of Eq. 27 for non-relativistic beams. This is because its derivation is based on a prior assumption, valid only for highly relativistic beams, that the monitor intercepts only and all the electric field lines of those beam particles within its length (see paragraph leading to Eq. 17.)

\section{AC Transformers with Hereward Feedback}

Electronic feedback may be used to extend the low frequency response of the ac beam current transformer. The need for such artificial enhancement may result from spatial constraints limiting the size of a magnetic toroid or from the expediency of utilizing a standard commercial device which may fall short of the required low frequency response. The former case often prevails in the low energy section of a Linac.(8) Space there is usually at a premium, yet high fidelity reproduction of a beam pulse several hundred microseconds in duration is required with minimal signal droop. The latter case exists in the Fermilab Booster where feedback means the difference between utilizing a standard commercial ac current transformer or resorting to a higher cost dc responding device. In the Booster satisfactory performance with less than $1 \%$ droop over a 35 millisecond beam current pulse is achieved.

A combined active/passive network which provides the benefits of Hereward feedback without sacrificing high frequency response due to amplifier bandwidth limitations is described by Unser.(1) A simplified circuit arrangement employing Hereward feedback is shown in Figure 4. Response of this simple circuit can be calculated in much the same manner as for the passive circuit in Fig. 2.

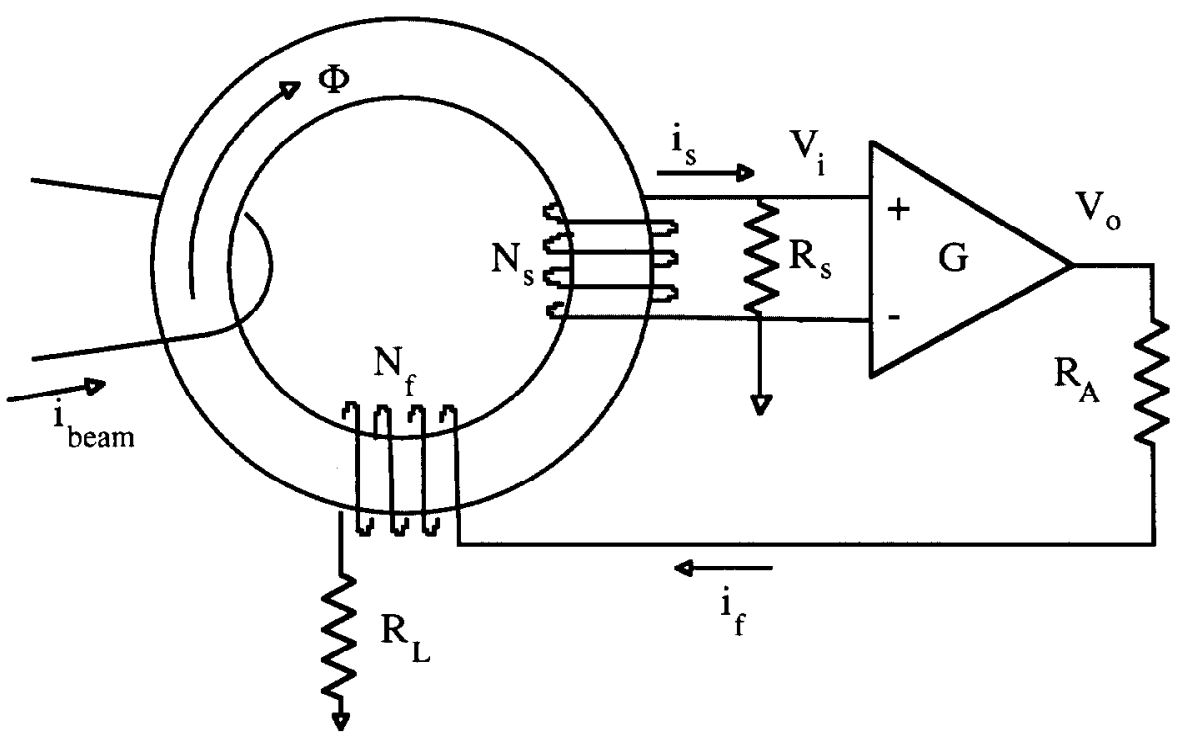

Figure 4. Beam current transformer circuit with Hereward feedback. 
Here $\Phi_{T}=L_{0} i_{b}+\frac{L_{s} i_{s}}{N_{s}}+\frac{L_{f} i_{f}}{N_{f}}, \quad V_{i}=-s N_{s} \Phi_{T}, \quad i_{s}=V_{i} / R_{s}, \quad$ and $V_{o}=G V_{i}=i_{f}\left(R_{A}+R_{L}\right)+s N_{f} \Phi_{T}$. Solving these equations simultaneously yields

$$
\frac{V_{o}}{i_{b}}=-\frac{s N_{s} G L_{0}}{1+s \tau}
$$

This is similar to the solution obtained for the circuit without feedback, except that $\tau$ now takes the more complicated form

$$
\tau_{H}=L_{0}\left(\frac{N_{s}^{2}}{R_{s}}+\frac{N_{f}^{2}}{R_{A}+R_{L}}+\frac{G N_{f} N_{s}}{R_{A}+R_{L}}\right)=\tau_{s}+\tau_{f}+\tau_{c}
$$

where $\tau_{c}=\frac{G L_{0} N_{f} N_{s}}{R_{A}+R_{L}}=\frac{G \sqrt{L_{s} L_{f}}}{R_{A}+R_{L}}$. In the usual case where the amplifier gain is large, the $\tau_{c}$ term dominates. Thus, the circuit is able to extend the low frequency response (increase the effective $\tau$ ) by a large factor, the order of magnitude of the amplifier gain. For frequencies $\omega \gg 1 / \tau_{c}$ the response simplifies to

$$
\frac{V_{o}}{i_{b}}=\frac{R_{A}+R_{L}}{N_{f}}
$$

Such performance enhancement appears attractive, but is not obtained without penalty. A look at the noise performance of the circuit reveals the trade-off. Imagine a noise voltage source in series with the amplifier input. $V_{i}$ becomes $V_{i}=-s N_{s} \Phi_{T}+V_{\text {noise }}$ and the circuit's solution is obtained in the same manner. The result appears in the form

$$
\frac{V_{o}}{V_{\text {noise }}}=\frac{G\left(1+s \tau_{f}\right)}{1+s \tau_{H}}
$$

where $\tau_{H}$ and $\tau_{\mathrm{f}}$ are as defined above and, for convenience, the beam current has been set to zero. At frequencies well above $1 / \tau_{f}$ this becomes $V_{o} / V_{\text {noise }}=N_{f} / N_{s}$. Feedback via the coupled windings suppresses noise amplification and the noise sensitivity is frequency independent. At frequencies well below $1 / \tau_{H}$ the noise sensitivity becomes $V_{o} / V_{\text {noise }}=G$. The noise appears at the output amplified by the full gain of the amplifier. This occurs 
because at such low frequencies there is effectively no coupling between the feedback and sense windings; the magnetic transformer no longer plays any role in the circuit operation. This is typically evidenced in such circuits by high sensitivity to amplifier drift and signal baseline wander that is difficult to control. These problems usually limit the maximum acceptable gain and the low frequency cutoff enhancement that is possible.

At the amplifier output, the signal to noise ratio is then

$$
\mathrm{SNR}=\frac{-\left(\frac{s N_{s} G L_{0}}{1+s \tau_{H}}\right) i_{b}}{\left(\frac{G\left(1+s \tau_{f}\right)}{1+s \tau_{H}}\right) V_{\text {noise }}}=-\frac{s L_{0} N_{s} i_{b}}{V_{\text {noise }}\left(1+s \tau_{f}\right)} .
$$

Above the frequency corresponding to $\tau_{f}$ (i.e., the natural time constant of the feedback winding circuit) the SNR is constant at $\operatorname{SNR}=\left(\frac{N_{s}}{N_{f}^{2}}\right)\left(\frac{i_{b}\left(R_{A}+R_{L}\right)}{V_{\text {noise }}}\right)$. Below that frequency the SNR degrades at $20 \mathrm{db}$ per decade, even though the signal sensitivity remains flat down to $1 / \tau_{H}$ ! This is depicted in Fig. 5. At $\omega=1 / \tau_{H}$, where signal roll-off begins, the signal to noise ratio has deteriorated by a factor equal to the feedback circuit gain $G$. The enhanced low frequency response is obtained at the expense of increased noise.

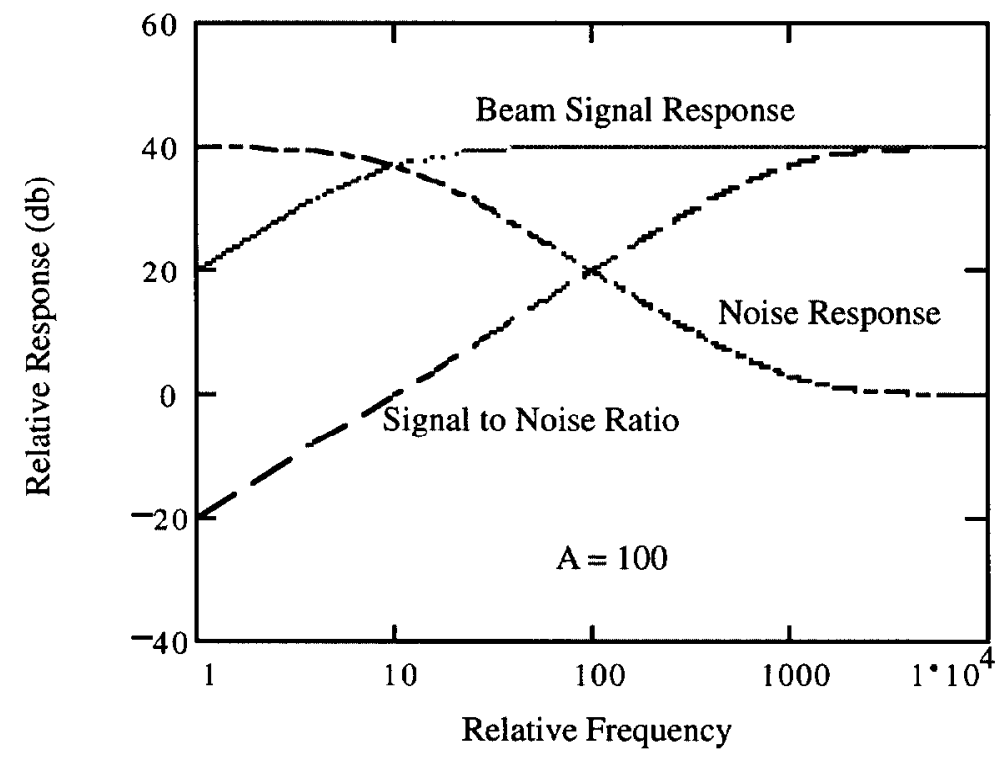

Figure 5. Response of transformer circuit with Hereward feedback, $A=100$. 


\section{WALL CURRENT MONITORS}

Devices known as wall current monitors are usually employed for measuring beam current signals with bandwidths greater than about $100 \mathrm{Mhz}$. They are able to resolve the temporal shape of beam bunches to better than 100 picoseconds, typical of those found in proton and other hadron accelerators.(9) A wall current monitors function as single turn transformer, but may be simply thought of as a monitoring resistance placed directly in the path of the wall currents. Typical transfer impedances for wall current monitors (i.e., output volts per unit beam current) are from several hundred milliohms to several ohms.

The art of wall current monitor design and construction involves smoothly channeling the wall currents on the inner surface of the beam tube through the monitoring resistance, tapping onto that resistance in a manner so as to yield a signal voltage insensitive to beam position, and properly controlling all shunt impedances; this, all with flat response over gigahertz bandwidths. A detailed discussion of wall current monitors has been written by Webber.(10)

\section{DIRECT CURRENT TRANSFORMERS}

Direct Current Transformers (DCTs) are able to provide accurate and high precision measurement of circulating beam currents over a dynamic range of $10^{5}$ or greater. Sensitivities of better than $1 \mu \mathrm{A}$ are achievable and DCTs built at Fermilab have operated over periods of years with baseline drifts of no more than $5 \mu \mathrm{A}$. A great deal of literature is available describing the operation and design details of DCTs.(11, 12, 13, 14, 15) High quality devices are commercially available.

Since the de beam current provides no time varying flux component to generate a signal by magnetic induction, an ac flux component is "brought to the beam' via the action of a magnetic modulator circuit. The operation of a magnetic modulator, and hence a DCT, is based on the non-linear characteristics of high quality tapewound magnetic cores. Figure 6 shows a simplified DCT magnetic modulator layout. Two toroidal cores are switched between flux saturation levels, first one polarity then the other, by counter-phased windings powered by an external source. In the absence of any dc beam current and to the extent that the two cores exhibit matched and symmetric B-H characteristics, sense windings of a common polarity around each core produce equal and opposite signals. The output of either winding is non-zero only during that time in which the flux in the cores is changing (i.e., not saturated.) The sum of the two sense winding signals is zero. This is depicted in Fig. 7a.

A dc beam current through the two cores biases each with flux of the same polarity, while the flux in one core due to the modulator drive remains out of phase with that in the second.(Fig. 7b) Each core reaches its saturation flux level at a 


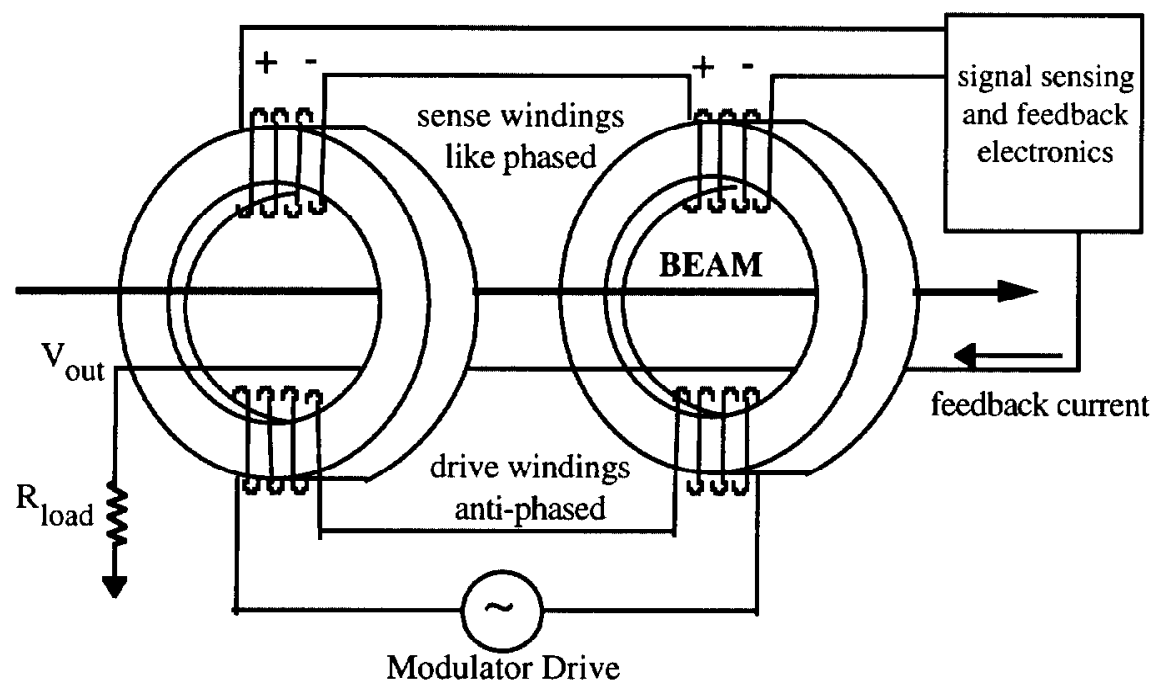

Figure 6. Magnetic modulator section of DC Transformer with flux nulling feedback.
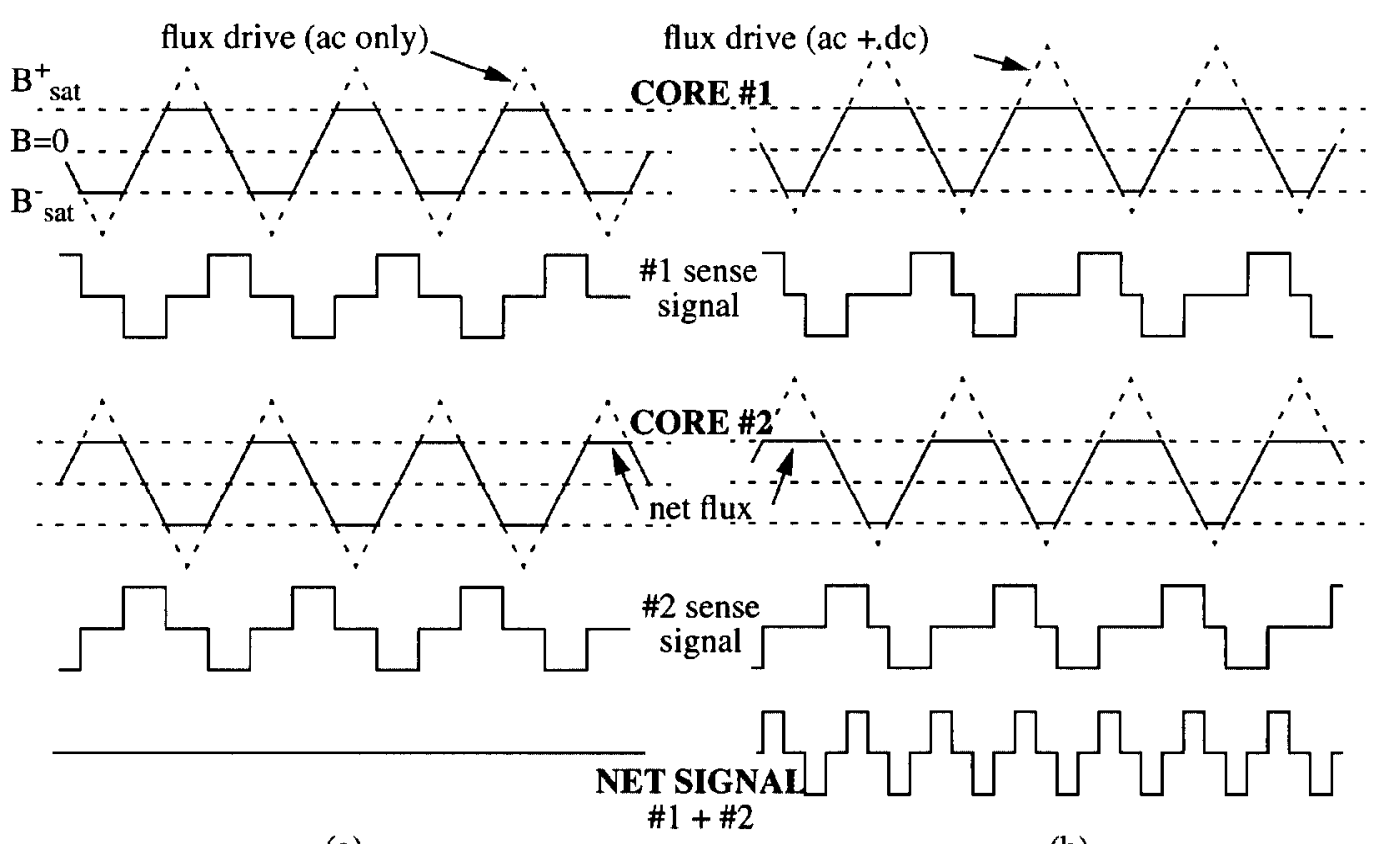

(a)

(b)

Figure 7. Magnetic modulator signals, (a) with no dc present and (b) with exaggerated dc presence. 
different point in the excitation cycle for one alternation than for the other alternation. The net result is a flux imbalance between the two cores, producing an output signal at even harmonics of the excitation frequency.

The magnetic modulator section of the DCT is essentially a magnetic 'mixer', translating dc signals to a different location in the frequency spectrum. Functioning as a sampling device, it will produce aliasing of signal frequencies greater than half the excitation frequency. For example, a modulator operating at $1 \mathrm{Khz}$ drive frequency will produce an identical output for a $1 \mathrm{Khz}$ beam current component as for an equal magnitude dc current. This problem is usually avoided in a beam current monitoring DCT by coupling the dc modulator with an ac transformer in a hybrid network with a crossover frequency well below one half the excitation frequency. DCTs are normally designed to operate in a feedback configuration as shown in Fig. 6. Current is forced through a feedback winding on the cores to oppose the beam current and maintain a dc flux null.

\section{REFERENCES}

1. Unser, K. B., "Toroidal AC and DC Current Transformers for Beam Intensity Measurements," Atomkernenergie - Kerntechnik, Vol. 47, 1985, No.1.

2. Borer, J. and Jung, R., "Diagnostics," presented at CERN Accelerator School on Antiprotons for Colliding Beam Facilities, October 11-21, 1984, Geneva, also CERN/LEP-BU/84-14.

3. Lambertson, G. R., "Electromagnetic Detectors," presented at Joint USCERN School on Particle Accelerators, October 20-26, 1988, Isola di Capri, also LBL-26075.

4. Siemann, R. H., "Bunched Beam Diagnostics," in Physics of Particle Accelerators, (1987 and 1988 U. S. Particle Accelerator School proceedings), AIP Conference Proceedings No. 184, ISBN 0-88318-384-6, 1989.

5. Halliday, D. and Resnick, R., Fundamentals of Physics, 3rd Ed.(John Wiley \& Sons, New York, 1988), Ch. 31, p. $714 \mathrm{ff}$.

6. Unser, K.B.,"Measuring Bunch Intensity, Beam Loss and Bunch Lifetime in LEP," presented at EPAC 90, Nice, France, June 12-16, 1990, also CERN/ SL/90-27 (BI).

7. Unser, K. B., "Design and Preliminary Tests of a Beam Intensity Monitor for LEP," 1989 IEEE Particle Accelerator Conference Proceedings, IEEE Catalog No. $89 \mathrm{CH} 2669-0$, pp.

8. Power, J. F., "Compact Cryogenic Toroid for Beam Current Measurements," submitted to Nuclear Particle Beam Technology Symposium, Monterey, CA, July 17-21, 1989, also LA-CP-89-383. 
9. C. D. Moore, et al., "Single Bunch Intensity Monitoring System Using an Improved Wall Current Monitor," 1989 IEEE Particle Accelerator Conference Proceedings, IEEE Catalog No. 89CH2669-0, pp. 1513-1515.

10. Webber, R. C., "Longitudinal Emittance: An Introduction to the Concept and Survey of Measurement Techniques, Including Design of a Wall Current Monitor," in Accelerator Instrumentation, (Proceedings of the First Annual Accelerator Instrumentation Workshop, 1989, Upton, New York), AIP Conference Proceedings No. 212, ISBN 0-88318-645-4, 1990, pp. 85-126.

11. Unser, K., "Beam Current Transformer with D.C. to $200 \mathrm{Mhz}$ Range," IEEE Trans. Nuc. Sci., NS-16, June, 1969, pp. 934-938.

12. Unser, K. B., "The Parametric Current Transformer, A Beam Current Monitor Developed for LEP," in Accelerator Instrumentation, (Proceedings of the Third Accelerator Instrumentation Workshop, 1991, CEBAF, Newport News, VA), AIP Conference Proceedings No. 252, ISBN 0-88318-934-8, 1992, also CERN SL/91-42 (BI).

13. Talman, R., "Beam Current Monitors," in Accelerator Instrumentation, (Proceedings of the First Annual Accelerator Instrumentation Workshop, 1989, Upton, New York), AIP Conference Proceedings No. 212, ISBN 088318-645-4, 1990, pp. 1-25.

14. Schutte, W., "Mechanical Design of the Beam Current Transformers for the HERA Proton Ring," 1991 Particle Accelerator Conference Proceedings, IEEE Catalog No. 91CH3038-7, pp.

15. Burtin, G., et al., "Mechanical Design, Signal Processing and Operator Interface of the LEP Beam Current Transformers," presented at EPAC 90, Nice, France, June 12-16, 1990, also CERN/SL/90-30 (BI). 